Notfall Rettungsmed 2017 $20: 61-62$

DOI 10.1007/s10049-016-0260-8

Online publiziert: 3 . Januar 2017

๑) Springer Medizin Verlag Berlin 2016

CrossMark
K. Wedler ${ }^{1,2} \cdot$ M. Machner ${ }^{3} \cdot$ J. Mersmann ${ }^{4} \cdot$ S. Schuster ${ }^{5,6} \cdot$ A. Pozniak $^{2} \cdot$ P. Jahn ${ }^{1} \cdot$ F. Walcher ${ }^{2}$

'Stabsstelle Pflegeforschung, Universitätsklinikum Halle (Saale), Halle (Saale), Deutschland ${ }^{2}$ BMBF-Projekt A.L.I.N.A., Klinik für Unfallchirurgie, Universitätsklinikum Magdeburg, Magdeburg, Deutschland

${ }^{3}$ Gesundheitsakademie, Charité - Universitätsmedizin Berlin, Berlin, Deutschland

${ }^{4}$ Konzern-Notaufnahmen, Mühlenkreiskliniken, Minden, Deutschland

${ }^{5}$ Evangelische Hochschule Nürnberg, Nürnberg, Deutschland

${ }^{6}$ Studienzentrale, Zentrale Notaufnahmen, Klinikum Fürth, Fürth, Deutschland

\section{Erratum zu: Entwicklungen und Perspektiven der Notfallpflege in Deutschland}

\section{Erratum zu:}

Notfall Rettungsmed (2016)

19: 540-547

DOI:10.1007/s10049-016-0212-3

Die Autoren machen auf einen Fehler unter der Überschrift „Angewandte Pflegewissenschaften Schwerpunkt Notfallpflege (B.Sc.) “ aufmerksam. So liegen die Kosten des Studiengangs „Angewandte Pflegewissenschaften " mit dem Profilschwerpunkt "Notfallpflege“ bei einem Semesterbeitrag von $315 €$ bei insgesamt $1890 €$. Dies wird hiermit berichtigt. Wir bitten diesen Fehler zu entschuldigen.

\section{Korrespondenzadresse}

\section{K. Wedler, BSc PGW, MHMM}

BMBF-Projekt A.L.I.N.A., Klinik für Unfallchirurgie, Universitätsklinikum Magdeburg

Magdeburg, Deutschland

katrin.wedler@med.ovgu.de 
Hier steht eine Anzeige.

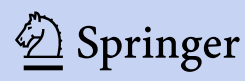

